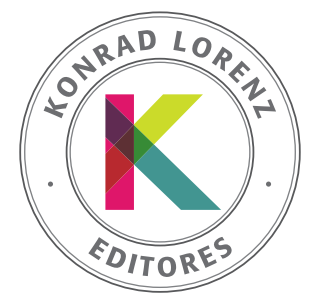

\title{
Compromiso e intención de abandono en adolescentes de selecciones nacionales argentinas: el papel de los agentes sociales y efectos del género
}

\author{
María Julia Raimundi a,b,d,*, Ignacio Celsi a,c, Carla Otero c, Vanina Schmidt a,c,d, \\ Isabel Castillo e
}

\author{
${ }^{a}$ Consejo Nacional de Investigaciones Científicas y Técnicas (CONICET), Argentina \\ ${ }^{b}$ Instituto de Psicología Básica, Aplicada y Tecnología (IPSIBAT), Universidad Nacional de Mar del Plata, Argentina \\ ' Universidad de Buenos Aires, Facultad de Psicología, Instituto de Investigaciones, Argentina \\ ' Universidad Abierta Interamericana, Facultad de Psicología y Relaciones Humanas, Argentina \\ e Universitat de València, Facultad de Psicología y Logopedia, España
}

Recibido el 5 de agosto de 2020; aceptado el 2 de marzo de 2021

\author{
PALABRAS CLAVE \\ Desarrollo deportivo, \\ alto rendimiento, \\ compromiso, \\ estilos interpersonales, \\ contexto social
}

\begin{abstract}
Resumen Introducción/objetivo. El objetivo de esta investigación es examinar el poder predictivo de la percepción de los estilos interpersonales de entrenadores/as, padres y madres en el compromiso e intención de abandono de adolescentes deportistas argentinos de alto rendimiento, considerando el papel del género en esta relación. Método. Participaron 234 adolescentes de entre 12 y 16 años $(M=14.48$, $D T=1.09)$ seleccionados para los Juegos Olímpicos de la Juventud -Buenos Aires 2018-, quienes cumplimentaron cuestionarios para la evaluación de las variables de interés. Resultados. Los varones perciben mayores niveles de compromiso que las mujeres y estas perciben mayor apoyo a la autonomía de la madre. El apoyo a la autonomía del entrenador tuvo mayor capacidad predictiva en el compromiso de los varones y en la intención de abandono del deporte de las mujeres. En ambos géneros, el efecto del apoyo a la autonomía del entrenador fue mayor que el de los padres. Conclusiones. Estos resultados muestran la importancia de los otros significativos en la participación deportiva y contribuyen al conocimiento de los factores que pueden favorecer el desarrollo positivo en deportistas adolescentes de alto rendimiento.
\end{abstract}

(c) 2021 Fundación Universitaria Konrad Lorenz. Este es un artículo Open Access bajo la licencia CC BY-NC-ND (http://creativecommons.org/licenses/by-nc-nd/4.0/).

\footnotetext{
* Autor para correspondencia.

Correo electrónico: juliaraimundi@gmail.com
} 


\section{KEYWORDS:}

Sport development, high-performance, engagement, interpersonal styles, social context

\section{Engagement and intentions of drop-out in adolescents of Argentinean national teams: The role of social agents and gender effects}

\begin{abstract}
Introduction/Objective. This research aims to examine the predictive power of perceived interpersonal styles of coaches, fathers and mothers on engagement and intentions to drop-out of Argentinean high-performance adolescent athletes considering the role of gender in this relationship. Method. A total of 234 adolescents aged $12-16$ years $(M=14.48, S D=1.09)$ selected for the Youth Olympic Games -Buenos Aires 2018- participated in the study, who answered questionnaires assessing the variables of interest. Results. Males perceived higher levels of engagement than females, and females perceived higher autonomy support from the mother. Coaches' autonomy support had greater predictive power on males' engagement and females' intentions to drop-out sport. In both genders, the effect of coach autonomy support was greater than parents. Conclusions. These results show the importance of significant others in sport participation and contribute to the knowledge of factors that may foster positive development in high-performance adolescence athletes.

(c) 2021 Fundación Universitaria Konrad Lorenz. This is an open access article under the CC BY-NC-ND license (http://creativecommons.org/licenses/by-nc-nd/4.0/).
\end{abstract}

El deporte de alto rendimiento implica el nivel más alto de realización deportiva y se distingue por una gran dedicación (tiempo, disposición, capacidad para la acción), necesaria para lograr los niveles de ejecución necesarios en el propósito de conseguir el rendimiento máximo, así como de alcanzar los mejores resultados a nivel internacional. Los adolescentes involucrados en los programas de desarrollo deportivo y de representación nacional están considerados como potenciales para alcanzar estos altos estándares (Swann et al., 2015). Diferentes estudios abordados desde el modelo de transiciones de carreras deportivas han mostrado que el éxito en la transición al alto rendimiento está vinculado al logro de una adaptación a los desafíos deportivos, psicosociales y académico-laborales que se les presentan en la nueva etapa (Stambulova \& Wylleman, 2019). Para esto es necesario el desarrollo no solo de habilidades y competencias psicológicas, sino también contar con factores del entorno que acompañen esta transición (Prato et al., 2020).

En esta línea, las variables motivacionales constituyen uno de los recursos personales relevantes a la hora de afrontar el proceso de transición al alto rendimiento (Chamorro et al., 2016). El compromiso con el deporte (engagement) se define como un proceso cognitivo-afectivo persistente y positivo, caracterizado por la creencia en la propia capacidad de lograr un nivel de rendimiento y conseguir metas (confianza), el deseo de invertir esfuerzo y tiempo en el logro de objetivos importantes para uno (dedicación), el vigor o vivacidad física, mental y emocional (energía) y la sensación de disfrute (entusiasmo) (Lonsdale et al., 2007). Esta variable motivacional constituye la esencia de la participación en este nivel competitivo y se vincula a consecuencias positivas para estos deportistas (e.g., Balk et al., 2019). Por esto resulta importante estar en capacidad de examinar las características que contribuyen, en este nivel de práctica, a favorecer un contexto de desarrollo positivo en el deporte adolescente.

La teoría de la autodeterminación (SDT, Ryan \& Deci, 2017) postula que el contexto social tiene una importancia central para la calidad de las experiencias, a través del rol que desempeñan los otros significativos. En el contexto del deporte, y específicamente en el deporte adolescente, los/as entrenadores/as, los padres y las madres constituyen agentes sociales relevantes en la experiencia de estos jóvenes (De Muynck et al., 2020).

Las conductas de los entrenadores pueden conceptualizarse en términos de dos estilos interpersonales: el apoyo a la autonomía y el estilo controlador (Vallerand \& Losier, 1999). El primero describe entrenadores que apoyan activamente las iniciativas de los deportistas y crean condiciones para que experimenten un sentido de elección y desarrollo personal, mientras que en el estilo controlador el entrenador actúa de manera coercitiva y autoritaria para imponer su forma de pensar y actuar (Bartholomew et al., 2010). La evidencia muestra que los climas en los que se apoya la autonomía tienden a ser más beneficiosos para la participación deportiva, ya que se relacionan con niveles altos de motivación intrínseca, generando mayor diversión en el deporte, vitalidad y autoestima, lo que fomenta el trabajo en equipo y produce una menor probabilidad de abandonar el deporte (e.g., González et al., 2016). En cambio, los climas con características controladoras se relacionan con conductas negativas o desadaptativas por parte de los deportistas: mayores índices de ansiedad y miedo al fracaso, menor autoestima, menor deportividad, mayor burnout y mayor probabilidad de abandono (e.g., Castillo et al., 2012).

El papel de los padres también constituye un aspecto relevante en el desarrollo de la carrera deportiva, desde la iniciación hasta la etapa de perfeccionamiento (e.g., Harwood \& Knight, 2015). Los padres de los adolescentes deportistas de alto rendimiento pueden funcionar como personas importantes para obtener consejos y ayuda en los momentos difíciles (e.g., bajo rendimiento, malos resultados, lesiones), propios en este nivel competitivo (Raimundi et al., 2019). Se ha encontrado que los adolescentes de alto rendimiento perciben un funcionamiento familiar más positivo que los de otros niveles (Raimundi et al., 2016). Aquellos jóvenes que perciben una implicación activa y apoyo por parte de sus padres poseen mayores niveles de diversión y compromiso en la práctica de su deporte, mientras que las conductas de presión o comportamientos directivos hacia los hijos conllevan a una mayor predisposición al abandono (e.g., De Muynck et al., 2020; Torregrossa et al., 2007). Por tanto, es relevante conocer el impacto que tienen estas variables familiares en las experiencias deportivas de los adolescentes de alto rendimiento. 
Diferentes estudios han mostrado que las variables del contexto familiar y el papel de los entrenadores tiene un efecto diferente en función del género de los deportistas (e.g., Álvarez et al., 2019; Amado et al., 2015; Koh \& Wang, 2015; Raimundi et al., 2019). Sin embargo, los resultados no son concluyentes debido a la heterogeneidad de los participantes en estos estudios (i.e., deportistas en edad de formación, alto rendimiento, deporte recreativo, etc.) y a la utilización de diferentes instrumentos de evaluación, sobre todo, para las medidas de los padres. Asimismo, hasta el momento no se han encontrado estudios que examinen conjuntamente la percepción de los deportistas respecto a los estilos interpersonales de entrenadores y padres considerando el género. Por tanto, el objetivo de este trabajo es estudiar el papel de la percepción de los estilos interpersonales de entrenadores y padres como predictores del compromiso y la intención de abandono en deportistas adolescentes de alto rendimiento, considerando el género en esta relación.

\section{Método}

\section{Tipo de estudio y diseño}

Estudio de estrategia asociativa de diseño no experimental transversal con muestreo por conveniencia.

\section{Participantes}

Participaron 234 adolescentes de entre 12 y 16 años $(M=14.48 ; D T=1.09)$, seleccionados para el equipo olímpico argentino de los Juegos Olímpicos de la Juventud. Se tomaron los géneros masculinos y femeninos desde una perspectiva binaria, en tanto los participantes se autopercibieron como de género masculino (47.90\%) y femenino cisgénero $(52.10 \%)$. Los deportes más practicados eran handball $(10.68 \%)$, vela $(10.26 \%)$, gimnasia artística $(8.98 \%)$, canotaje $(7.69 \%)$, natación $(7.26 \%)$, básquetbol (6.84\%), triatlón (5.56\%), tiro deportivo (5.13\%), y taekwondo $(4.27 \%)$, entre otros. El promedio de práctica era de 7.46 años $(D T=3.06)$ y el de entrenamiento de 16.18 horas semanales $(D T=8.69)$.

\section{Instrumentos}

Características sociodemográficas. Se evaluó el género, la edad, el deporte practicado, los años y las horas de práctica semanal del deporte.

Apoyo a la autonomía del entrenador. Se utilizó el Spanish Sport Climate Questionnaire (S-SCQ, Balaguer et al., 2009) adaptado al español para Argentina (Raimundi, Pérez-Gaido, et al., 2021), compuesto por 15 ítems (e.g., "Confío mucho en mi entrenador/a"). Las respuestas se recogen en una escala Likert de 1 (totalmente en desacuerdo) a 5 (totalmente de acuerdo). La escala original y su adaptación poseen indicadores adecuados de validez y confiabilidad.

Estilo interpersonal controlador del entrenador. Se utilizó la escala Controlling Coach Behaviors Scale (CCBS; Bartholomew et al., 2010) adaptada al español para Argen- tina (Raimundi, Pérez-Gaido, et al., 2021), compuesta por 15 ítems (e.g., "Mi entrenador/a intenta controlar lo que hago en mi tiempo libre"). Las respuestas se recogen en una escala Likert de 1 (totalmente en desacuerdo) a 5 (totalmente de acuerdo). La escala original y su adaptación en español (Castillo et al., 2014) poseen adecuados indicadores de validez y confiabilidad.

Apoyo a la autonomía de los padres. Se utilizó la subescala de apoyo a la autonomía de ambos padres del Perceptions of Parental Scales (POPS; Robbins, 1994) en su adaptación al español (Castillo \& Balaguer, 2008). La subescala está compuesta por 18 ítems (nueve para cada versión) (e.g., "Mi madre/padre me permite decidir las cosas por mí mismo/a"), y las respuestas se recogen en una escala Likert de 1 (totalmente en desacuerdo) a 5 (totalmente de acuerdo). La subescala ha mostrado adecuados indicadores de validez y confiabilidad (Niemiec et al., 2009; Robbins, 1994).

Compromiso con el deporte. Se utilizaron los 16 ítems del Athlete Engagement Questionnaire (AEQ; Lonsdale et al., 2007) adaptado para Argentina (Raimundi, Celsi, et al., 2021) que evalúa las cuatro dimensiones (cuatro ítems por factor) del compromiso: confianza ("Creo que puedo conseguir mis metas deportivas"), dedicación ("Lo doy todo para alcanzar mis metas deportivas"), energía ("Me siento lleno/a de energía cuando practico mi deporte") y entusiasmo ("Disfruto con mi deporte"). Las respuestas se recogen en una escala Likert de 1 (casi nunca) a 5 (casi siempre). Para el presente estudio se utilizaron las puntuaciones de cada dimensión y la puntuación total (compromiso). La escala original y sus adaptaciones poseen excelentes propiedades psicométricas.

Intención de abandono del deporte. Se utilizaron tres ítems del cuestionario desarrollado por Quested et al. (2013), con cinco opciones de respuesta, a fin de evaluar el grado de acuerdo respecto de las intenciones de dejar el deporte la próxima temporada (e.g., "Tengo intención de abandonar el deporte cuando acabe esta temporada"). La versión original y su adaptación en español poseen adecuados indicadores de validez y confiabilidad.

\section{Procedimiento}

La presente investigación contó con la aprobación del Comité de Ética de la Facultad de Psicología de la Universidad de Buenos Aires. Se incluyó el protocolo de cuestionarios en la hoja de ruta de evaluaciones a cumplimentar por los deportistas seleccionados por el Ente Nacional de Alto Rendimiento Deportivo (ENARD), con la finalidad de formar parte del proyecto olímpico -Buenos Aires 2018-, con consentimiento escrito de sus padres. Los adolescentes respondieron de forma grupal y anónima, con asistencia de cuatro miembros del equipo de investigación a cargo. Todos los entrenadores y los coordinadores del proyecto recibieron un informe, a modo de devolución, con los principales resultados obtenidos para cada grupo de deportistas.

\section{Análisis de datos}

Como análisis preliminares, se realizaron dos análisis de componentes de varianza (con el programa SAS 9.2), utili- 
zando los procedimientos de mínimos cuadrados y máxima verosimilitud. Los resultados mostraron que los errores residuales son iguales o casi iguales para ambos procedimientos en todas las variables, por tanto, se asume que la distribución de los datos es lineal, normal y homocedástica (Searle et al., 1992), lo que permite utilizar estadísticos paramétricos para los siguientes análisis.

Se calcularon los descriptivos, las correlaciones de Pearson y los coeficientes alfa de Cronbach. Las diferencias por género se calcularon mediante la prueba $t$. Se realizaron análisis de regresión lineal múltiple por método de selección de pasos sucesivos para examinar el poder predictivo de los estilos interpersonales sobre el compromiso y la intención de abandono. Para la interpretación del tamaño del efecto $\left(r\right.$ y $\left.f^{2}\right)$ se tomaron los criterios de Cohen (1992) (pequeño = $.10 / .02$; mediano $=.30 / .15$; grande $=.50 / .35$, respectivamente). Todos estos análisis se realizaron con el SPSS v.23.

\section{Resultados}

En la tabla 1 se presentan los estadísticos descriptivos, los índices de consistencia interna para cada una de las variables y los valores de los errores residuales obtenidos en los análisis de componentes varianza. Los alfas de Cronbach fueron buenos, a excepción de la intención de abandono. No obstante, un valor alfa de .60 puede considerarse aceptable (Hair et al., 2006) en escalas con un número reducido de ítems, como es el caso. Los descriptivos mostraron con respecto a la media escalar, que los adolescentes perciben niveles altos de compromiso y de apoyo a la autonomía del entrenador y de ambos padres, mientras que perciben bajos niveles de estilo controlador del entrenador e intención de abandono.

Los varones presentaron mayores puntajes, de modo que se diferencian significativamente de las mujeres en compromiso (total) y en las dimensiones de confianza y energía, mientras que las mujeres puntuaron significativamente más alto en apoyo a la autonomía de la madre (tabla 2).

Entre los varones, el compromiso y todas sus dimensiones correlacionaron significativamente y de manera positiva con el apoyo a la autonomía del entrenador, mientras que solo el apoyo a la autonomía del padre se asoció significativamente con dedicación. La intención de abandono se relacionó negativamente con el apoyo a la autonomía del entrenador. Entre las mujeres, el compromiso se asoció positivamente con el apoyo a la autonomía del entrenador y de la madre, y negativamente con el estilo controlador del entrenador. Respecto de las dimensiones, la confianza se asoció positivamente con el apoyo a la autonomía del entrenador y negativamente con el estilo controlador, mientras que la energía se asoció negativamente con dicho estilo controlador. Asimismo, la energía, la dedicación y el entusiasmo se asociaron positivamente con el apoyo a la autonomía de la madre. La intención de abandono se asoció a ambos estilos interpersonales del entrenador, de manera positiva con el apoyo a la autonomía y negativamente con el estilo controlador. Los tamaños del efecto de todas las correlaciones fueron medianos, a excepción del apoyo a la autonomía del entrenador y del compromiso entre los varones, y dicho apoyo a la autonomía con intención de abandono entre las mujeres que tuvieron efectos grandes (tabla 3).

A fin de analizar la contribución de los estilos interpersonales en el compromiso e intención de abandono, se realizaron análisis de regresión para el género femenino y masculino por separado (tabla 4). Se comprobaron que los valores del índice de Durbin-Watson fueron adecuados -entre 1.5 y 2.5- (Pardo \& Ruiz, 2005) y los estadísticos de colinealidad indicaron valores aceptables de inflación de la varianza y del índice de tolerancia -menores a 10- (Hair et al., 2006). Para los varones, la variable con capacidad predictiva para el compromiso y sus dimensiones fue el apoyo a la autonomía del entrenador. Los porcentajes de varianza explicada para los modelos varió entre $11 \%$ y $26 \%$, con un tamaño del efecto mediano y grande. Para las mujeres, el apoyo a la autonomía de la madre resultó predictor del compromiso y de las dimensiones de energía, dedicación y entusiasmo, mientras que el estilo controlador el entrenador lo fue para confianza. Los porcentajes de varianza oscilaron entre $4 \%$ y $14 \%$ con tamaños del efecto pequeños. La variable que presentó mayor capacidad predictiva para la intención de abandono, en ambos grupos, fue el apoyo a la autonomía del entrenador. Ahora bien, para los varones el modelo explicó un $7 \%$ de la varianza de

Tabla 1 Descriptivos de las variables del estudio para la muestra total

\begin{tabular}{|c|c|c|c|c|c|c|}
\hline & \multirow{2}{*}{ Media (DT) } & \multirow{2}{*}{ Asimetría } & \multirow{2}{*}{ Curtosis } & \multirow{2}{*}{$\alpha$} & \multicolumn{2}{|c|}{ Errores residuales } \\
\hline & & & & & Varcomp I & GLM \\
\hline Confianza & $4.17(0.59)$ & -0.80 & 0.73 & .71 & 9069.16 & 9069.16 \\
\hline Energía & $4.24(0.57)$ & -0.52 & -0.43 & .75 & 6576.91 & 6576.91 \\
\hline Dedicación & $4.51(0.53)$ & -1.41 & 2.14 & .78 & 7141.22 & 7141.22 \\
\hline Entusiasmo & $4.65(0.43)$ & -1.25 & 0.91 & .73 & 4678.93 & 4678.93 \\
\hline Compromiso (total) & $4.39(0.41)$ & -0.63 & 0.34 & .87 & 9494.09 & 9494.09 \\
\hline Intención de abandono & $1.40(0.66)$ & 2.10 & 4.95 & .60 & 1339.56 & 1339.56 \\
\hline Apoyo autonomía entrenador/a & $4.16(0.53)$ & -0.88 & 1.05 & .88 & 14022 & 14022.17 \\
\hline Estilo controlador entrenador/a & $2.11(0.67)$ & 0.25 & -0.72 & .87 & 23166 & 23165.85 \\
\hline Apoyo autonomía madre & $3.95(0.54)$ & -0.69 & 0.62 & .72 & 5212.14 & 5212.14 \\
\hline Apoyo autonomía padre & $3.88(0.55)$ & -0.49 & 0.39 & .70 & 5253.15 & 5253.15 \\
\hline
\end{tabular}

Nota. Rango de las variables 1-5, DT = desviación típica, $\alpha=$ alfa de Cronbach, Varcomp = procedimiento de mínimos cuadrados; $\mathrm{GLM}=$ procedimiento de máxima verosimilitud. 
Tabla 2 Comparación del compromiso, la intención de abandono y la percepción de los agentes sociales en función del género

\begin{tabular}{|c|c|c|c|c|c|}
\hline & $\begin{array}{c}\text { Masculino }(n=112) \\
\text { Media }(D T)\end{array}$ & $\begin{array}{c}\text { Femenino }(n=122) \\
\text { Media }(D T)\end{array}$ & $t$ & $p$ & $r$ \\
\hline Confianza & $4.30(0.52)$ & $4.05(0.63)$ & 3.20 & $.002^{* *}$ & .37 \\
\hline Energía & $4.38(0.54)$ & $4.12(0.58)$ & 3.49 & $.001^{* *}$ & .40 \\
\hline Dedicación & $4.57(0.50)$ & $4.46(0.56)$ & 1.52 & .128 & .18 \\
\hline Entusiasmo & $4.68(0.44)$ & $4.62(0.42)$ & 0.96 & .337 & .11 \\
\hline Compromiso (total) & $4.48(0.41)$ & $4.31(0.40)$ & 3.11 & $.002^{* *}$ & .36 \\
\hline Intención de abandono & $1.36(0.64)$ & $1.44(0.67)$ & -0.89 & .373 & .11 \\
\hline Apoyo autonomía entrenador/a & $4.18(0.48)$ & $4.13(0.56)$ & 0.75 & .449 & .09 \\
\hline Estilo controlador entrenador/a & $2.19(0.67)$ & $2.04(0.66)$ & 1.67 & .095 & .19 \\
\hline Apoyo autonomía madre & $3.87(0.53)$ & $4.02(0.54)$ & -2.13 & $.034^{*}$ & .25 \\
\hline Apoyo autonomía padre & $3.89(0.49)$ & $3.87(0.59)$ & 0.29 & .771 & .03 \\
\hline
\end{tabular}

Nota. $r=$ tamaño del efecto

${ }^{*} p<.05 ;{ }^{* *} p<.01$;

la variable con un tamaño del efecto pequeño, mientras que para las mujeres el modelo explicó un $27 \%$ con un tamaño del efecto grande (tabla 4).

\section{Discusión}

El objetivo de este trabajo fue estudiar el poder predictivo de los estilos interpersonales de los/as entrenadores/as, los padres y las madres y el papel del género, en el compromiso e intención de abandono en deportistas adolescentes de alto rendimiento seleccionados para competir en los Juegos Olímpicos de la Juventud.

Los resultados mostraron que los varones perciben niveles significativamente más altos de compromiso que las mujeres. Estudios europeos que examinaron el compromiso deportivo no encontraron diferencias significativas en función del género en el puntaje total, aunque sí en las mismas dimensiones encontradas en el presente estudio: confianza y energía (e.g., Babić et al., 2015). Tradicionalmente, las situaciones deportivas han estado más adaptadas a la capacidad de los varones que a la de las mujeres (Moreno-Murcia et al., 2009), lo cual ha generado contextos que pueden no ser del todo satisfactorios o ha limitado el desarrollo de características fundamentales para el rendimiento deportivo, como, por ejemplo, la confianza. Según Alfaro (2004), la falta de equidad en el camino de las mujeres al alto rendimiento se da en el ámbito escolar (se generan menos expectativas en sus profesores), familiar (se presta menos atención a los intereses deportivos de las hijas) y en el ámbito profesional, en el que claramente existe una diferencia en los recursos económicos para el deporte femenino. Esta situación puede generar que los varones perciban mayor entusiasmo y confianza y se involucren más en la realización del deporte, mientras que estas diferencias ubican a las mujeres en un lugar de menor percepción de eficacia, dado que se internalizan estos estereotipos (Wasend \& LaVoi, 2019).

Con relación a la percepción de los estilos interpersonales de los agentes sociales, se encontró que las mujeres perciben mayor apoyo a la autonomía de la madre que los varones. En este sentido, respecto a la comunicación familiar, también se encontró en adolescentes de selecciones argentinas que las mujeres percibían menor restricción en la comunicación con la madre, es decir, que pueden contar con la madre para contarles cuestiones de su intimidad en mayor medida que los varones (Raimundi et al., 2016).

Respecto a las correlaciones, se encontró que, para los varones, el compromiso se asocia con el apoyo a la autonomía del entrenador y solo la dedicación se asocia, a su vez,

Tabla 3 Correlaciones entre las variables del estudio en función del género

\begin{tabular}{|c|c|c|c|c|c|c|c|c|}
\hline & \multicolumn{4}{|c|}{ Entrenador/a } & \multirow{2}{*}{\multicolumn{2}{|c|}{$\begin{array}{c}\text { Madre } \\
\text { Apoyo autonomía }\end{array}$}} & \multirow{2}{*}{\multicolumn{2}{|c|}{$\begin{array}{c}\text { Padre } \\
\text { Apoyo autonomía }\end{array}$}} \\
\hline & \multicolumn{2}{|c|}{ Apoyo autonomía } & \multicolumn{2}{|c|}{ Estilo controlador } & & & & \\
\hline & Masc. & Fem. & Masc. & Fem. & Masc. & Fem. & Masc. & Fem. \\
\hline Confianza & $.34^{* *}$ & $.22^{* *}$ & -.11 & $-.22^{*}$ & .07 & .12 & .01 & .06 \\
\hline Energía & $.40^{* *}$ & .09 & -.01 & $-.19^{*}$ & .14 & $.39^{* *}$ & .04 & .17 \\
\hline Dedicación & $.48^{* *}$ & .07 & -.15 & -.11 & .09 & $.22^{*}$ & $.20^{*}$ & .10 \\
\hline Entusiasmo & $.46^{* *}$ & .16 & -.14 & -.14 & .17 & $.23^{*}$ & .11 & .11 \\
\hline Compromiso (total) & $.51^{* *}$ & $.19^{*}$ & -.12 & $-.23^{*}$ & .14 & $.32^{* *}$ & -.15 & -.14 \\
\hline Intención de abandono & $-.28^{*}$ & $-.52^{* *}$ & .04 & $.29^{* *}$ & .11 & .15 & -.07 & .04 \\
\hline
\end{tabular}

Nota. Masc. $=$ Masculino, Fem. $=$ Femenino

$p<.05 ;{ }^{* *} p<.01$ 
Tabla 4 Poder predictivo de las variables de los agentes sociales en el compromiso e intención de abandono en función del género

\begin{tabular}{|c|c|c|c|c|c|c|c|c|c|c|c|c|}
\hline \multirow{2}{*}{$\begin{array}{l}\text { Variable } \\
\text { criterio }\end{array}$} & \multirow[b]{2}{*}{ Grupo } & \multicolumn{5}{|c|}{ Modelo } & \multirow{2}{*}{$\begin{array}{c}\text { Variables } \\
\text { predictoras }\end{array}$} & \multirow[b]{2}{*}{ B } & \multirow[b]{2}{*}{$t$} & \multirow[b]{2}{*}{$p$} & \multirow[b]{2}{*}{$\mathrm{T}$} & \multirow[b]{2}{*}{ FIV } \\
\hline & & $R^{2}$ & $\begin{array}{c}R^{2} \\
\text { corregida }\end{array}$ & DW & $F$ & $f^{2}$ & & & & & & \\
\hline \multirow{2}{*}{ Confianza } & Masculino & .12 & .11 & 1.91 & 14.12 & .14 & AA-E & .34 & 3.75 & $<.001$ & 1.00 & 1.00 \\
\hline & Femenino & .05 & .04 & 1.58 & 6.15 & .05 & $E C-E$ & -.22 & -2.48 & .015 & 1.00 & 1.00 \\
\hline \multirow{2}{*}{ Energía } & Masculino & .16 & .15 & 2.08 & 20.69 & .19 & $A A-E$ & .40 & 4.54 & $<.001$ & 1.00 & 1.00 \\
\hline & Femenino & .15 & .14 & 1.92 & 20.84 & .18 & AA-M & .39 & 4.56 & $<.001$ & 1.00 & 1.00 \\
\hline \multirow{2}{*}{ Dedicación } & Masculino & .23 & .22 & 2.23 & 30.93 & .30 & AA-E & .48 & 5.56 & $<.001$ & 1.00 & 1.00 \\
\hline & Femenino & .05 & .04 & 2.15 & 5.99 & .05 & AA-M & .22 & 2.44 & .016 & 1.00 & 1.00 \\
\hline \multirow{2}{*}{ Entusiasmo } & Masculino & .21 & .21 & 2.19 & 29.02 & .27 & $A A-E$ & .46 & 5.38 & $<.001$ & 1.00 & 1.00 \\
\hline & Femenino & .05 & .04 & 2.39 & 6.32 & .05 & $A A-M$ & .23 & 2.51 & .013 & 1.00 & 1.00 \\
\hline \multirow{2}{*}{$\begin{array}{l}\text { Compromiso } \\
\text { (total) }\end{array}$} & Masculino & .26 & .26 & 2.15 & 38.51 & .35 & AA-E & .51 & 6.21 & $<.001$ & 1.00 & 1.00 \\
\hline & Femenino & .11 & .10 & 1.98 & 13.72 & .12 & AA-M & .32 & 3.71 & $<.001$ & 1.00 & 1.00 \\
\hline \multirow{2}{*}{$\begin{array}{l}\text { Intención } \\
\text { de abandono }\end{array}$} & Masculino & .08 & .07 & 1.80 & 9.06 & .07 & $A A-E$ & -.28 & -2.34 & .003 & 1.00 & 1.00 \\
\hline & Femenino & .27 & .27 & 1.63 & 43.39 & .37 & AA-E & -.52 & -5.92 & $<.001$ & 1.00 & 1.00 \\
\hline
\end{tabular}

Nota. $\mathrm{AA}=$ apoyo a la autonomía, $\mathrm{EC}=$ estilo controlador, $\mathrm{E}=$ entrenador $/ \mathrm{a}, \mathrm{M}=$ madre, $\mathrm{P}=$ padre, $\mathrm{DW}=\mathrm{Durbin}-$ Watson, $\mathrm{T}=$ índice de tolerancia, FIV = factor de inflación de la varianza

con el apoyo del padre. Respecto a esta última relación, se ha mostrado que es el padre el que pasa más tiempo y se involucra más en la actividad deportiva de los hijos varones que en la de sus hijas (Bean et al., 2014). Para las mujeres, el compromiso se asocia a ambos estilos interpersonales del entrenador y al apoyo a la autonomía de la madre. Estos resultados son consistentes con la literatura: las mujeres tienden a ser más permeables a la influencia de las variables del contexto que los varones (Raimundi et al., 2019; Schmidt et al., 2008). Ahora bien, la dimensión del compromiso que se asoció a las conductas de los entrenadores entre las mujeres fue la confianza, mientras que las demás se asociaron con el apoyo de la madre. La SDT postula que las mujeres suelen percibir un locus de causalidad más autónomo (Ryan \& Deci, 2017), por tanto, para ellas los agentes sociales en su conjunto (entrenadores y padres) podrían favorecer el compromiso y la motivación en el deporte. Resulta interesante observar que en el presente estudio el apoyo a la autonomía del padre no se ha asociado a ninguna de las variables estudiadas (a excepción de la ya comentada entre dedicación y apoyo a la autonomía del padre entre los varones), mientras que el apoyo a la autonomía de la madre se ha asociado exclusivamente a las variables de compromiso en mujeres. Este resultado va en consonancia con lo encontrado por Álvarez et al. (2019), quienes constataron que el apoyo a la autonomía de la madre se asociaba con un menor burnout de sus hijas, de manera que se amortigua el posible efecto negativo que los padres podían tener al no sentirse apoyadas por ellos.

Los modelos de regresión mostraron que las experiencias en el deporte varían en función del género. Para los varones, el apoyo a la autonomía del entrenador es la variable que resultó mejor predictora del compromiso y de sus dimensiones, mientras que, para las mujeres, fue el apoyo a la autonomía de la madre. En deportistas españoles se mostró que el papel de la madre adquiere mayor relevancia que el del padre para la protección del burnout (Álvarez et al., 2019). Asimismo, si bien el núcleo primordial del en- torno afectivo está constituido por ambos padres, estudios en niños y adolescentes muestran que la madre todavía es la principal encargada de las tareas de cuidado (Monteiro et al., 2019) y confidente de los problemas de los adolescentes en América Latina y el Caribe (Cepal, 2004). Por tanto, se puede considerar que, en familias iberoamericanas, el papel de la madre adquiere mayor relevancia para el ajuste psicosocial de los adolescentes, en población general y también cuando se trata de deportistas.

La intención de abandono, para ambos géneros, se relacionó con los estilos interpersonales de los entrenadores, pero no con los de los padres. Específicamente, el apoyo a la autonomía fue la variable con mayor capacidad predictiva, de manera que fue mayor la asociación para las mujeres que para los varones. Esto refuerza el papel fundamental de los entrenadores en el desarrollo de la motivación en la medida en que su comportamiento, sus valores y su filosofía tienen consecuencias cognitivas, emocionales y conductuales en sus deportistas (Duda, 2013).

En cuanto a la capacidad predictiva más alta para las mujeres, diversos estudios muestran el papel diferencial de los entrenadores en las experiencias de los deportistas considerando el género. En adolescentes deportistas olímpicos se encontró que los varones percibían mayores conductas de apoyo por parte de los entrenadores (Koh \& Wang, 2015) y que el clima motivacional de implicación en la tarea (con características similares al apoyo a la autonomía) de los entrenadores predijo el compromiso y la diversión en deportistas varones, pero no en mujeres (Torregrossa et al., 2011). Si se trata de clases de educación física los resultados difieren. Por ejemplo, se encontró que el feedback de los profesores es más beneficioso en generar afecto positivo en las mujeres (Mouratidis et al., 2008) y también son ellas las que suelen responder mejor a esa comunicación (Nicaise et al., 2006).

Ahora bien, es importante destacar que para los varones de este estudio los entrenadores pueden funcionar más como un facilitador del compromiso, mientras que en el 
caso de las mujeres como un factor protector del abandono. Se encontró que las mujeres tienden a ver más controladores a sus entrenadores, con menor apoyo a la autonomía y apoyo social que los varones, todo lo cual impacta más sobre el decremento de la motivación intrínseca (Fernández et al., 2018). Tales diferencias pueden explicarse en tanto se aprecie que el deporte es aún un contexto de logro mayoritariamente masculino, en el que los practicantes son, en su mayoría, de este género, y en el que los entrenadores, las entrenadoras y la formación que reciben en las federaciones deportivas también está a cargo de ellos (LaVoi et al., 2019). Aun con progresos -como el identificado en el estudio de Barriopedro et al. (2016) respecto de la inserción laboral de los deportistas olímpicos-, el deporte ha sido un ámbito que se ha abierto menos que otros a un desarrollo equitativo (Leiter et al., 2020). Podría pensarse que esta formación está basada u orientada tomando en cuenta más los procesos que favorecen la implicación y el rendimiento de los varones. Es decir, los entrenadores están más acostumbrados a entrenar en la rama masculina y pueden identificarse más con estos, por lo cual es posible que sus conductas coincidan más con las conductas favorecedoras de climas positivos para los varones, mientras que en el caso de la rama femenina estas mismas conductas son solo protectoras del abandono. Si bien los resultados de este estudio muestran qué conductas pueden proteger del abandono deportivo, será importante continuar indagando cómo los agentes sociales pueden favorecer el compromiso deportivo para las adolescentes.

Los resultados del presente estudio mostraron que los estilos interpersonales de los entrenadores son predictores más potentes que los de los padres tanto para el compromiso como para la intención de abandono de los deportistas adolescentes de alto rendimiento. Los padres, como agentes de socialización fundamentales en la vida de los adolescentes, son los principales proveedores de oportunidades para que sus hijos se involucren en el deporte, ya que son los que ofrecen un ambiente favorecedor de incentivo y constituyen fuentes de apoyo instrumental y emocional (Raimundi et al., 2016). A pesar de esto, los diferentes agentes sociales de la vida de un deportista (en este caso, padres y entrenadores) pueden influir en diferentes niveles de generalidad: los padres afectarían a un nivel global, puesto que son una referencia a lo largo de los diferentes contextos de la vida de los jóvenes, mientras que los entrenadores representarían la figura jerárquica de referencia al determinar los valores y las metas en el interior de los equipos que dirigen (e.g., Barbee, 2020).

Como limitaciones se puede señalar que se trata de un estudio transversal, por lo que no es posible establecer relaciones de causalidad entre las variables. Futuras investigaciones con diseños longitudinales podrían examinar con mayor profundidad el compromiso de los jóvenes deportistas de alto rendimiento y la influencia del contexto social a lo largo de su carrera. La información se obtuvo mediante cuestionarios, por lo que futuros estudios podrían utilizar algún método cualitativo (e.g., observación) que complemente la información obtenida.

Algunos estudios mostraron que las madres tienen más influencia en la introducción de deportes tales como gimnasia y natación, independientemente del género del hijo, mientras que es el padre el que tiene un rol más preponderante cuando se trata de fútbol (Baxter-Jones \& Maffulli,
2003). Por tanto, podría resultar de interés contemplar las características del deporte estudiado (individual o colectivo, la cultura e historia de esos deportes, el rol de los estereotipos de género, etc.) respecto a los estilos interpersonales de los padres y el papel del género de los entrenadores en su interacción con el de los deportistas, teniendo en cuenta la representación desigual en la proporción de entrenadoras y entrenadores (Leiter et al., 2020).

Llegar al alto rendimiento implica un largo proceso de entrenamiento, esfuerzo y dedicación. Por esta razón el compromiso constituye una base fundamental para mejorar el rendimiento y obtener beneficios de esa participación (Torregrossa et al., 2016). Así, pues, los resultados de este estudio pueden ser relevantes a la hora de desarrollar estrategias desde las federaciones deportivas y los profesionales del deporte, con el fin de promover trayectorias integrales de los deportistas adolescentes en el camino hacia el alto rendimiento. El acompañamiento de los agentes sociales es clave en esta etapa de desarrollo, por tanto, se pueden diseñar programas de capacitación dirigidos a entrenadores y padres con el objetivo de reflexionar acerca de las conductas actuales de estos agentes sociales y brindar recursos específicos (e.g., estrategias de comunicación, comportamientos adaptativos en entrenamientos y torneos) para apoyar la autonomía de los adolescentes, evitar la presión y, con esto, favorecer el compromiso y el disfrute del deporte.

\section{Agradecimientos}

A los deportistas que participaron, a sus entrenadores y al Ente Nacional de Alto Rendimiento Deportivo (ENARD) que permitió la realización del estudio. A los organismos que financiaron el proyecto: Consejo Nacional de Investigaciones Científicas y Técnicas (Conicet Res. 4883/2015), Secretaría de Ciencia y Técnica de la Universidad de Buenos Aires (Proyecto UBACyT 20020130100434) y a las Ayudas de movilidad del Vicerrectorado de Internalización y Cooperación de la Universitat de València que permitieron estancias de intercambio y trabajo conjunto.

\section{Nota de las/os autoras/es}

A lo largo del manuscrito se utiliza el genérico "entrenadores" y "padres" para designar tanto a varones como mujeres, bien sean entrenadoras o entrenadores, o bien madres o padres.

\section{Referencias}

Alfaro, E. (2004). El talento psicomotor y las mujeres en el deporte de alta competición. Revista de Educación, 335, 127-151.

Álvarez, O., Castillo, I., \& Moreno-Pellicer, R. (2019). Transformational parenting style, autonomy support, and their implications for adolescent athletes' burnout. Psychosocial Intervention, 27(1), 91-100. https://doi.org/10.5093/pi2019a7

Amado, D., Sánchez-Oliva, D., González-Ponce, I., Pulido-González, J., \& Sánchez-Miguel, P. (2015). Incidence of parental support and pressure on their children's motivational processes towards sport practice regarding gender. PLOS ONE, 10(6). https://doi.org/10.1371/journal.pone.0128015 
Babić, V., Sarac, J., Missoni, S., \& Sindik, J. (2015). Athletic engagement and athletic identity in top Croatian sprint runners. Collegium Antropologicum, 39(3), 521-528.

Balaguer, I., Castillo, I., Duda, J., \& Tomás, I. (2009). Análisis de las propiedades psicométricas de la versión española del Cuestionario de Clima en el deporte. Revista de Psicología del Deporte, 18(1), 73-83.

Balk, Y. A., de Jonge, J., Geurts, S. A. E., \& Oerlemans, W. G. M. (2019). Antecedents and consequences of perceived autonomy support in elite sport: a diary study linking coaches' off-job recovery and athletes' performance satisfaction. Psychology of Sport and Exercise, 44, 26-34. https://doi.org/10.1016/j. psychsport.2019.04.020

Barbee, J. (2020). Association of coach, peer, and parent-initiated motivational climate with burnout and engagement (Tesis doctoral). East Carolina University. http://hdl.handle. net/10342/8563

Barriopedro, M., Muniesa, C., \& López de Subijana, C. (2016). Gender perspective at the Spanish olympic athletes' employment. Cuadernos de Psicología del Deporte, 16(1), 339-349. https:// revistas.um.es/cpd/article/view/254691

Bartholomew, K., Ntoumanis, N., \& Thøgersen-Ntoumani, C. (2010). The controlling interpersonal style in a coaching context: development and initial validation of a psychometric scale. Journal of Sport \& Exercise Psychology, 32(2), 193-216.

Baxter-Jones, A., \& Maffulli, N. (2003). Parental influence on sport participation in elite young athletes. Journal of Sports Medicine and Physical Fitness, 43(2), 250-255.

Bean, C., Fortier, M., Post, C., \& Chima, K. (2014). Understanding how organized youth sport may be harming individual players within the family unit: a literature review. International Journal of Environmental Research and Public Health, 11(10), 10226-10268. https://doi.org/10.3390/ijerph111010226

Castillo, I., \& Balaguer, I. (6-8 de noviembre de 2008). Clima motivacional creado por los entrenadores e implicación familiar como predictores de la vitalidad subjetiva en jóvenes jugadoras de tenis: Análisis de los mecanismos motivacionales. II Congreso de la Sociedad Iberoamericana de Psicología del Deporte, Torrelavega, España.

Castillo, I., González, L., Fabra, P., Mercé, J., \& Balaguer, I. (2012). Estilo interpersonal controlador del entrenador, frustración de las necesidades psicológicas básicas, y burnout en futbolistas infantiles. Cuadernos de Psicología del Deporte, 12(1), 143-146. https://doi.org/10.4321/S1578-84232012000100014

Castillo, I., Tomás, I., Ntoumanis, N., Bartholomew, K., Duda, J., \& Balaguer, I. (2014). Psychometric properties of the Spanish version of the Controlling Coach Behaviors Scale in the sport context. Psicothema, 26(3), 409-414. https://doi.org/10.7334/ psicothema2014.76

Cepal (2004). La juventud en Iberoamérica. Tendencias y urgencias. https://www.cepal.org/es/publicaciones/2785-la-juventud-iberoamerica-tendencias-urgencias

Chamorro, J., Sánchez-Oliva, D., Pulido-González, J., \& Amado, D. (2016). Will I play in the elite? Expectations to become professional and their relationship with motivation in young football players. Retos, 29, 153-156.

Cohen, J. (1992). A power primer. Psychological Bulletin, 112(1), 155-159. https://doi.org/10.1037/0033-2909.112.1.155

De Muynck, G. de, Morbée, S., Soenens, B., Haerens, L., Vermeulen, O., Vande Broek, G., \& Vansteenkiste, M. (2020). Do both coaches and parents contribute to youth soccer players' motivation and engagement? An examination of their unique (de)motivating roles. International Journal of Sport and Exercise Psychology, 1-19. https://doi.org/10.1080/1612197X.2020.1739111

Duda, J. (2013). The conceptual and empirical foundations of Empowering Coaching ${ }^{\mathrm{TM}}$ : setting the stage for the PAPA project.
International Journal of Sport and Exercise Psycchology, 11(4), 37-41. https://doi.org/10.1080/1612197X.2013.839414

Fernández, A., García-Arabehety, M., Celsi, I., Tourn, S., \& Raimundi, M. J. (3-5 de octubre de 2018). Clima motivacional creado por los entrenadores: Diferencias en la percepción de deportistas adolescentes de Buenos Aires (Argentina) en función del género, deporte y categoría. VII Congreso Iberoamericano de Psicología del Deporte, Santiago, Chile.

González, L., García-Merita, M., Castillo, I., \& Balaguer, I. (2016). Young athletes' perceptions of coach behaviors and their implications on their well and ill-being over time. Journal of Strenght and Conditioning Research, 30(4), 1147-1154.

Hair, J., Black, W., Babin, B., Anderson, R., \& Tatham, R. (2006). Multivariate data analysis (6. ${ }^{\mathrm{a}}$ ed.). Prentice Hall.

Harwood, C., \& Knight, C. (2015). Parenting in youth sport: a position paper on parenting expertise. Psychology of Sport and Exercise, 16, 24-35. https://doi.org/10.1016/j.psychsport.2014.03.001

Koh, K., \& Wang, C. (2015). Gender and type of sport differences on perceived coaching behaviours, achievement goal orientations and life aspirations of youth Olympic games Singaporean athletes. International Journal of Sport and Exercise Psychology, 13(2), 91-103. https://doi.org/10.1080/1612197X.2014.932820

LaVoi, N., McGarry, J., \& Fisher, L. (2019). Final thoughts on women in sport coaching: frighting the war. Women in Sport and Physical Activity Journal, 27(2), 136-140. https://doi.org/10.1123/ wspaj.2019-0030

Leiter, E., Raimundi, M. J., \& Todarello, F. (2020). Las actitudes sexistas ambivalentes de entrenadores y entrenadoras y su relación la percepción del clima motivacional que crean en sus equipos deportivos. Educación Física y Ciencia, 22(4), 1-10.

Lonsdale, C., Hodge, K., \& Jackson, S. (2007). Athlete engagement: II. Development and initial validation of the Athlete Engagement Questionnaire. International Journal of Sport Psychology, 38, 471-492.

Monteiro, L., Torres, N., \& Salinas-Quiroz, F. (2019). Preditores do envolvimento paterno numa amostra de famílias portuguesas. O papel das crenças parentais. Suma Psicológica, 26(2), 94-102. https://doi.org/10.14349/sumapsi.2019.v26.n2.5

Moreno-Murcia, J., Cano, F., González-Cutre, D., Cervelló, E., \& Ruiz, L. (2009). Flow disposicional en salvamento deportivo: una aproximación desde la teoría de la autodeterminación. Revista de Psicología del Deporte, 18(1), 23-35.

Mouratidis, A., Vansteenkiste, M., Lens, W., \& Sideridis, G. (2008). The motivating role of positive feedback in sport and physical education: evidence for a motivational model. Journal of Sport and Exercise Psychology, 30(2), 240-268. https://doi. org/10.1123/jsep.30.2.240

Nicaise, V., Cogérino, G., Bois, J., \& Amorose, A. (2006). Students' perceptions of teacher feedback and physical competence in physical education classes: gender effects. Journal of Teaching in Physical Education, 25(1), 36-57. https://doi.org/10.1123/ jtpe.25.1.36

Niemiec, C., Ryan, R., \& Deci, E. (2009). The path taken: consequences of attaining intrinsic and extrinsic aspirations in post-college life. Journal of Research in Personality, 43(3), 291306. https://doi.org/10.1016/j.jrp.2008.09.001

Pardo, A., \& Ruiz, M. (2005). Análisis de datos con SPSS 13 Base. McGraw-Hill.

Prato, L., Ramis, Y., \& Torregrossa, M. (2020). Cultural transition and sport migration in elite sport: a meta-synthesis. Cultura, Ciencia y Deporte, 15(45), 387-400.

Quested, E., Ntoumanis, N., Viladrich, C., Haug, E., Ommundsen, Y., Van Hoye, A., Mercé, J., Hall, H., Zourbanos, N., \& Duda, J. (2013). Intentions to drop-out of youth soccer: a test of the basic needs theory among European youth from five countries. 
International Journal of Sport and Exercise Psycchology, 11(4), 395-407. https://doi.org/10.1080/1612197X.2013.830431

Raimundi, M. J., Molina, M. F., Schmidt, V., \& Hernández-Mendo, A. (2016). Family functioning profiles and character strengths in young elite athletes from Argentina. International Journal of Sport and Exercise Psychology, 17(1), 32-51. https://doi. org/10.1080/1612197X.2016.1189949

Raimundi, M. J., Molina, M. F., \& Hernández-Mendo, A. (2019). Escala de Promoción de Desafíos por parte de los padres en deportistas adolescentes de alto rendimiento. Liberabit: Revista Peruana de Psicología, 25(1), 57-70. https://doi.org/10.24265/ liberabit.2019.v25n1.05

Raimundi, M. J., Celsi, I., Iglesias, D., García-Arabehety, M., \& Schmidt, V. (2021). Engagement in youth sport: validity and reliability of the Athlete Engagement Questionnaire in athletes from Argentina. Manuscrito en preparación.

Raimundi, M. J., Pérez-Gaido, M., Celsi, I., \& Castillo, I. (2021). La evaluación de la percepción de las conductas de los entrenadores en deportistas adolescentes de Argentina. Manuscrito en preparación.

Robbins, R. (1994). An assessment of perceptions of parental autonomy support and control: Child and parent correlates (Tesis doctoral). University of Rochester.

Ryan, R., \& Deci, E. (2017). Self-determination theory. Basic psychological needs in motivation, development and wellness. The Guilford Press.

Schmidt, V., Messoulam, N., \& Molina, M. F. (2008). Autoconcepto académico en adolescentes de escuelas medias: presentación de un instrumento para su evaluación. Revista Iberoamericana de Evaluación Psicológica, 1, 81-106.

Searle, S., Casella, G., \& McCulloch, C. (1992). Variance components. John Wiley \& Sons.
Stambulova, N., \& Wylleman, P. (2019). Psychology of athletes' dual careers: a state-of-the-art critical review of the European discourse. Psychology of Sport and Exercise, 42, 74-88. https:// doi.org/10.1016/j.psychsport.2018.11.013

Swann, C., Moran, A., \& Piggott, D. (2015). Defining elite athletes: issues in the study of expert performance in sport psychology. Psychology of Sport and Exercise, 16, 3-14. https://doi. org/10.1016/j.psychsport.2014.07.004

Torregrossa, M., Cruz, J., Sousa, C., Viladrich, C., Villamarín, F., García-Mas, A., \& Palou, P. (2007). La influencia de padres y madres en el compromiso deportivo de futbolistas jóvenes. $R e$ vista Latinoamericana de Psicología, 39(2), 227-237.

Torregrossa, M., Viladrich, C., Ramis, Y., Azócar, F., Latinjak, A., \& Cruz, J. (2011). Efectos en la percepción del clima motivacional generado por los entrenadores y compañeros sobre la diversión y el compromiso. Diferencias en función de género. Revista de Psicología del Deporte, 20(1), 243-255.

Torregrossa, M., Chamorro, J., \& Ramis, Y. (2016). Transición de junior a sénior y promoción de carreras duales en el deporte: una revisión interpretativa. Revista de Psicología Aplicada al Deporte y al Ejercicio Físico, 1(6), 1-11. https://doi. org/10.5093/rpadef2016a6

Vallerand, R., \& Losier, G. (1999). An integrative analysis of intrinsic and extrinsic motivation in sport. Journal of Applied Sport Psychology, 11(1), 142-169. https://doi.org/10.1080/ 10413209908402956

Wasend, M., \& LaVoi, N. (2019). Are women coached by women more likely to become sport coaches? Head coach gender and female collegiate athletes' entry into the coaching profession. Women in Sport and Physical Activity Journal, 27(2 Special Issue), 85-93. https://doi.org/10.1123/wspaj.2018-0043 